\title{
ON REPRESENTATIONS OF THE HOLOMORPH OF ANALYTIC GROUPS
}

\author{
DONG HOON LEE
}

\begin{abstract}
We completely characterize those complex analytic groups whose holomorph groups admit finite-dimensional faithful complex analytic representations.
\end{abstract}

Let $G$ be a complex analytic group, and let $\operatorname{Aut}(G)$ denote the group of all analytic automorphisms of $G$, endowed with its natural structure of a complex Lie group. The semidirect product $G$ ( $) \operatorname{Aut}(G)$ with respect to the natural action of $\operatorname{Aut}(G)$ on $G$ is called the holomorph of $G$. In [3], G. Hochschild has shown that if $G$ is faithfully representable (that is, $G$ admits a faithful finite-dimensional analytic representation) and if the maximum nilpotent normal analytic subgroup of $G$ is simply connected, then the holomorph of $G$ is faithfully representable. The main purpose of this paper is to give an intrinsic characterization of those complex analytic groups whose holomorphs are faithfully representable. The following is the main result:

THEOREM. Let $G$ be a faithfully representable complex analytic group. Then the holomorph of $G$ is faithfully representable if and only if $G$ satisfies one of the following:

(i) The maximum nilpotent normal analytic subgroup $N$ of $G$ is simply connected;

(ii) $G=G^{\prime}$;

(iii) $G / G^{\prime}$ is a 1-dimensional complex torus.

We note here that since $G$ is faithfully representable, the commutator group $G^{\prime}$ is closed by a result of Goto (see [3, Chapter XVIII, Theorem 4.5]).

For the most part, we make use of results and techniques of earlier work by Hochschild [2, 3], and also by Hochschild and Mostow on representations and representative functions of Lie groups.

Preliminary results on $R[G]$. Here we gather some of the results on representations and representative functions of Lie groups for later use. Let $G$ be a complex analytic group. For any complex-valued function $f$ on $G$ and $x \in G$, define the left translate $x f$ and the right translate $f x$ by $(x f)(y)=f(y x),(f x)(y)=f(x y), y \in G$. A continuous function $f: G \rightarrow \mathbf{C}$ is called a representative function if the complex linear space spanned by the left translates $x f$, where $x$ ranges over $G$, is finite

Received by the editors August 31, 1984.

1980 Mathematics Subject Classification. Primary 22E10; Secondary 22E45.

Key words and phrases. Holomorph, representative functions, reductive groups, unipotent groups. 
dimensional. The representative functions on $G$ form a $\mathbf{C}$-algebra which we denote by $R[G]$. Let $\phi$ be a complex analytic representation of $G$ in a finite-dimensional linear space, and let $[\phi]$ denote the set of all complex-valued functions on $G$ of the form $\tau \circ \phi$, where $\tau$ is a linear functional on the algebra of all linear endomorphisms of $V$. The set $[\phi]$ is a finite-dimensional subspace of $R[G]$ and is invariant under left and right translations by elements of $G$. The group $\operatorname{Aut}(G)$ acts naturally on $R[G]$ by composition $(f, \alpha) \rightarrow f \circ \alpha$ for $f \in R[G]$ and $\alpha \in \operatorname{Aut}(G)$. The $R[G]$ also admits a comultiplication $\gamma: R[G] \rightarrow R[G] \otimes R[G]$ with which $R[G]$ becomes a Hopf algebra. The antipode of this Hopf algebra is the map $f \rightarrow f^{\prime}$, where $f^{\prime}(x)=f\left(x^{-1}\right)$. If $f \in R[G]$ and $x, y \in G$, then $f(x y)=\sum_{j} g_{j}(x) h_{j}(y)$, where $\gamma(f)=\sum_{j} g_{j} \otimes h_{j}$.

Suppose now $G$ is a faithfully representable complex analytic group, and assume that $G / G^{\prime}$ is reductive. (We recall [4] that a complex analytic group $H$ is called reductive if it is faithfully representable and if every finite-dimensional complex analytic representation of $H$ is semisimple.) Then $R[G]$ is finitely generated as a C-algebra, and $G$ in this case is a semidirect product $G=K P$, where $K$ is the radical of $G^{\prime}$ and $P$ is a reductive complex analytic group (see [5, Theorem 5.2]). Let $S$ be a finite-dimensional bi-stable subspace of $R[G]$ whose elements, together with the constants, generate $R[G]$, and let $\phi$ be the representation of $G$ by left translations on $S$. Then $\phi$ is faithful, and $\phi(G)$ is an algebraic subgroup of the group of all linear automorphisms of $S$. Since every complex analytic representation of $G$ induces a unipotent representation of $K$ by Lie's Theorem, it follows that an analytic representation $\xi$ of $\phi(G)$ is rational if and only if $\xi=\sigma \circ \phi^{-1}$ for some complex analytic representation $\sigma$ of $G$. Note that the affine algebra of polynomial functions of $\phi(G)$ consists of functions of the form $f \circ \phi^{-1}$ with $f \in R[G]$. It is also clear that $\phi(K)$ is the unipotent radical of $\phi(G)$ and $\phi(P)$ is a maximal linear reductive subgroup of the algebraic group $\phi(G)$. Transporting the complex affine algebraic group structure of $\phi(G)$ to $G$ via $\phi^{-1}, G$ can be endowed with the structure of a complex affine algebraic group such that the polynomial functions on $G$ are precisely the elements of $R[G]$. In this case, $K$ is the unipotent radical and $P$ is a maximal linear reductive algebraic subgroup of the algebraic group $G$. For $\alpha \in \operatorname{Aut}(G)$, we have $f \circ \alpha \in R[G]$ whenever $f \in R[G]$. Hence, $\operatorname{Aut}(G)$ coincides with the group of all algebraic group automorphisms of the algebraic group $G$.

Proof of theorem. Before we prove the theorem, we first observe the following: Assume that $G / G^{\prime}$ is reductive, and let $G=K P$ be a semidirect decomposition where $K$ is the radical of $G^{\prime}$ and $P$ is a maximal reductive subgroup of $G$. By what we have discussed above, $G$ can be given the structure of a complex affine algebraic group such that $K$ is the unipotent radical of $G$. Since $P$ is reductive, its Lie algebra is reductive in the usual sense, and this implies that the commutator subgroup $P^{\prime}$ of $P$ is semisimple, and that $P=A P^{\prime}$, where $A$ is the connected component of 1 of the center $Z(P)$ of $P$. Since $P^{\prime}$ is faithfully representable, its center (and hence $A \cap P^{\prime}$ ) is finite. Noting that $G / K \cong P$ and $G^{\prime} / K \cong P^{\prime}$, we see that $G / G^{\prime} \cong$ $(G / K) /\left(G^{\prime} / K\right) \cong P / P^{\prime} \cong A /\left(A \cap P^{\prime}\right)$, and hence $\operatorname{dim}(Z(P))=0$ (resp. $\operatorname{dim}(Z(P))=1$ ) if and only if $G=G^{\prime}$ (resp. $G / G^{\prime}$ is isomorphic to the 1-dimensional complex torus). 
Now we are ready to prove our theorem. Suppose that $G$ (s) $\operatorname{Aut}(G)$ is faithfully representable, and assume that condition (i) of the theorem does not hold. We will first show that $G / G^{\prime}$ is reductive, and that the natural action of $\operatorname{Aut}(G)$ on $R[G]$ is locally finite. (That is, each orbit $f \circ \operatorname{Aut}(G)$ spans a finite-dimensional subspace of $R[G]$.)

Let $\tilde{\rho}$ be a faithful analytic representation of $G$ (s) $\operatorname{Aut}(G)$, and let $\rho$ denote the restriction of $\tilde{\rho}$ to $G$. For $f \in[\rho]$, choose a function $\tilde{f} \in[\tilde{\rho}]$ such that $\tilde{f} \mid G=f$. For any $\alpha \in \operatorname{Aut}(G)$, the translate $\left(1, \alpha^{-1}\right) f(1, \alpha)$ coincides with $f \circ \alpha$ on $G$. Since $f$ is a representative function on $G$ ( ) $\operatorname{Aut}(G)$, it follows that $[\rho] \circ \operatorname{Aut}(G)$ spans a finitedimensional linear space. Let $\mathscr{A}$ be the largest sub-Hopf algebra of $R[G]$ that is stable under the natural action of $\operatorname{Aut}(G)$ and that is locally finite as an $\operatorname{Aut}(G)$ module. The sub-Hopf algebra generated by the set $[\rho] \circ \operatorname{Aut}(G)$ is clearly contained in $\mathscr{A}$, because it is $\operatorname{Aut}(G)$-invariant and the action of $\operatorname{Aut}(G)$ on the sub-Hopf algebra is locally-finite. Since $[\rho]$ separates the points of $G, \mathscr{A}$ separates the points of $G$.

Next we show that $G / G^{\prime}$ is reductive. For this, we follow arguments used in the proof of Theorem 5 of [2] with some modification. Suppose that $G / G^{\prime}$ is not reductive. Thus there exists a closed subgroup $L$ of $G$ such that $G^{\prime} \leqslant L$ and $L / G^{\prime}$ is the maximum torus of the abelian group $G / G^{\prime}$. Then $G / L$ is a (nontrivial) vector group. We can find a closed normal subgroup $H$ of $G$ such that $L \leqslant H$ and $G / H \cong \mathbf{C}^{1}$. Thus $G$ splits over $H$, and we can write $G=H \cdot V$ (semidirect product), where $V$ is a 1-dimensional vector subgroup of $G$. Since the nilpotent analytic group $N$ is not simply connected, $N$ contains a 1-dimensional central torus $T$. We identify $V$ with the complex field $\mathbf{C}$, and $T$ with the multiplicative group of nonzero complex numbers. For $c \in \mathbf{C}$, define $\alpha_{c}: G \rightarrow G$ by $\alpha_{c}(x)=x \exp (c \pi(x))$, where $\pi: G \rightarrow V$ is the projection. Clearly, the $\alpha_{c}$ 's are analytic automorphisms of $G$. Pick a function $f \in[\rho]$ such that $f$ is not constant on $T$, and choose a basis $g_{1}, g_{2}, \ldots, g_{m}$ for the space spanned by the right translates $f \cdot G$ of $f$. Then we have

$$
f x=\sum_{j} f_{j}(x) g_{j}
$$

for some $f_{1}, f_{2}, \ldots, f_{m} \in R[G]$. For $u \in V$, and $c \in \mathbf{C}$,

$$
f \circ \alpha_{c}(u)=f((\exp (c u)) u)=\sum_{j} f_{j}(\exp (c u)) g_{j}(u) .
$$

If $z$ denotes the identity map on the group $T$, then the representative functions on $T$ are polynomials of the form $\sum_{i} c_{i} z^{i}$ with $c \in \mathbf{C}$. Thus, if $f_{j}^{\prime}$ denotes the restriction of $f_{j}$ to $T$, then we have $f_{j}^{\prime}=\Sigma_{i} c_{i j} z^{i}$ with complex coefficients $c_{i j}$. Hence for each $c \in \mathbf{C}$, $f \circ \alpha_{c}(u)=\sum_{i, j} c_{i j} \exp (i c u) g_{j}(u)$. Since $[\rho] \circ \operatorname{Aut}(G)$ spans a finite-dimensional space, the functions $f \circ \alpha_{c}, c \in \mathbf{C}$, all lie in a finite-dimensional space of $R[G]$, and hence we must have $c_{i j}=0$, for $i>0$ and for all $j$. This shows that the functions $f_{j}^{\prime}$ are all constant. But for $t \in T, f(t)=(f t)(1)=\sum_{j} f_{j}(t) g_{j}(1)$, and it follows that $f$ is constant on $T$, which contradicts the choice of $f$. Thus, $G / G^{\prime}$ is reductive.

Now $G / G^{\prime}$ is reductive, and hence by what we have observed before, $G$ may be viewed as a complex affine algebraic group with $R[G]$ being identified as the affine 
algebra of polynomial functions on $G$. Since the sub-Hopf algebra $\mathscr{A}$ of $R[G]$ separates the points of $G$, we have $\mathscr{A}=R[G]$ by [1, Theorem 6.6]; that is, $R[G]$ is locally finite as an $\operatorname{Aut}(G)$-module. Hence, $G$ is a conservative complex affine algebraic group in the sense of [6]. Let $G=K P$ be the decomposition given in the beginning of our proof. By [6, Theorem 3.2], either the connected component $Z$ of 1 in the center of $G$ is a unipotent subgroup or else the dimension of $Z(P)$ is at most 1. But since we assumed that the maximum nilpotent normal analytic subgroup $N$ of $G$ is not simply connected, $Z$ cannot be a vector group, and hence $Z$ is not unipotent. It follows that the dimension of $Z(P)$ is at most 1 , showing that either $G=G^{\prime}$ or $G / G^{\prime}$ is isomorphic to a 1 -dimensional torus. Hence we obtain the condition (ii) or (iii).

Next we prove the converse. Suppose $N$ is simply connected. Then $G$ (S) $\operatorname{Aut}(G)$ is faithfully representable by Hochschild [3]. Suppose now that the condition (ii) or (iii) holds. In particular, $G / G^{\prime}$ is reductive in either case, and hence $G$ may be given a structure of an affine algebraic group with $R[G]$ being the affine algebra of polynomial functions on $G$. As we have observed before, the condition (ii) or (iii) is equivalent to the condition $\operatorname{dim} Z(P) \leqslant 1$. Hence by [6, Theorem 3.2], $G$ is a conservative algebraic group, and thus the action of $\operatorname{Aut}(G)$ is locally-finite. Let $\phi$ be a finite-dimensional faithful analytic representation of $G$, and let $W$ denote the linear subspace spanned by $[\phi] \circ \operatorname{Aut}(G)$. Since $[\phi]$ is finite-dimensional, so is $W$. Define a representation $\sigma$ of $G$ (S) $\operatorname{Aut}(G)$ on the linear space $W$ by $\sigma(x, \alpha)(w)=$ $x\left(w \circ \alpha^{-1}\right)$, for $(x, \alpha) \in G$ (S) $\operatorname{Aut}(G)$, and $w \in W$. Clearly, $\sigma$ is an analytic representation of $G$ ( ) $\operatorname{Aut}(G)$. Since $W$ separates the points of $G, \sigma$ is faithful. The proof of the theorem is complete.

\section{REFERENCES}

1. G. Hochschild, Introduction to affine analytic groups, Holden-Day, San Francisco, Calif., 1971.

2. , Representative action of the automorphisms complex analytic groups, J. Algebra 37 (1975), 296-301.

3. $333-336$. On representing analytic groups with their automorphisms, Pacific J. Math. 78 (1978),

4. The structure of Lie groups, Holden-Day, San Francisco, Calif., 1965.

5. G. Hochschild and G. D. Mostow, Representations and representative functions of Lie groups. III, Ann. of Math. (2) 70 (1959), 85-100.

6. __ A A _ Atomorphisms of affine algebraic groups, J. Algebra 13 (1969), 535-543.

Department of Mathematics and Statistics, Case Western Reserve University, Cleveland, OHIO 44106 